\title{
Editorial
}

\section{Family doctors and patients: is effective nutrition interaction possible?}

This supplement contains the papers and a summary of the discussions at the second international meeting on the topic of nutrition work in general medical practice. Throughout the meeting participants used the words 'family physician', 'primary care physician' and 'general practitioner' (GP) as meaning essentially the same basic specialty of medicine.

The first workshop 'Nutritional Attitudes and Practices of Primary Care Physicians' was held in Heelsum, near Wageningen, the Netherlands, in December 1995 and published in the American Journal of Clinical Nutrition 18 months later (Supplement to June issue (no 6), 1997). That meeting had invited speakers from the Netherlands, UK, USA, Australia, Belgium, Brazil, Denmark, Germany, Italy and Norway.

This second meeting was held at the same place, Heelsum, the Netherlands on December 14-16 1998 and the proceedings are now published here 5 months later. The speed of publication was achieved by prompt submission of manuscripts and fast-track editing with full use of modern electronic technology. Invited speakers at the second meeting were from the Netherlands, USA, UK, Australia, Canada, Denmark, New Zealand, Spain and Sweden.

Between the two major meetings a shorter intermediate workshop was held at the XVI International Nutrition Congress, Montreal, Canada, August 1997 on the same theme, nutrition in the work of the primary care physician.

The organisers and the participants at these workshops believe this topic is one of the biggest missed opportunities for converting modern nutritional knowledge, the results of all the nutrition research into practical behaviours. It deserves the focus of these dedicated workshops - and a third major workshop will be held in 2001. We believe that these meetings are leading to operational research, guidelines (hard copy and software) are greater use of nutrition advice in medical practice.

Most of the conclusions of the first workshop on nutrition in family medicine have been confirmed and often amplified at this second workshop, namely:

- General practitioners are highly trusted,

- An important percentage of consultations involved a (potential) nutrition component,

- But doctors do not bring nutrition into their interaction with the patient as often they could,

- Barriers have been identified,

- Materials to help doctors with nutrition work do exist and others are being developed,

- Secondary and tertiary prevention is the main place for nutrition advice in general practice,

- Obesity is difficult to treat,

- GPs are not public health officers. Public health nutrition differs from individual nutrition advice,

- Doctors have to distil simplified principles, essentials of dietetics,
- Collaboration with dieticians happens sometimes but there is potential for more.

In this second workshop there were some changes of emphasis:

(i) Obesity has already become a bigger problem, but if doctors cannot do something about it they run away from this prime nutritional disorder.

(ii) Computers are being used by more GPs in their consultations with patients (not just in the office for billing). This opportunity means that preparation of nutrition software for primary care physicians to use is a priority.

(iii) The structure of general practice in different countries makes a difference to the type of nutrition work possible. Where GPs have a stable, official list of patients and where they are the gatekeepers for specialised medical services, doctors can get to know their patients better, and their families, and have more occasions for talking about nutrition.

(iv) Perceived barriers to nutrition action previously reported from a thorough study of Dutch primary care physicians - lack of time, insufficient skills have been confirmed in GP trainees. But the modelling research (see Hiddink's paper) is showing that predisposing factors and driving forces are more important than barriers (in willingness to try to treat obesity). It is also emerging that very little nutrition skill training is included in GP training, although the trainees would nearly all like to learn about general nutrition and especially disease-related nutrition (see Maiburg's paper).

(v) Patients will be more likely to respond to nutrition advice if the doctor first deals with the problems that worry them most (see Wasson's paper). The 'stages of change' concept is useful so that doctors can concentrate their advice where response is most likely. The interaction model (see can Woerkum's paper) gives the patients more feeling of shared ownership of dietetic advice.

(vi) Individual nutrition advice and public health dietary principles overlap in a doctor's practice. Public health nutrition campaigns and the impact of new government reports would get better reinforcement if GPs could be briefed - even consulted - before the press launch.

(vii) In the UK family physicians are able to delegate provision of dietetic details to practice nurses and health visitors who are part of the same team and work in the same premises. The doctor can abbreviate his/her nutritional advice to main principles and also discuss dietetic policies and new nutritional information with their colleagues in the team. 
(viii) There has been a lot of confusion about nutrition and food safety in recent years. Media hypes, exaggerated food scares, controversies, self-styled media experts, direct and indirect food advertising contrast sadly with standardised, universal evidence-based information about drugs. GPs are not as convinced by the evidence about the real benefits of dietary change as they are about, for example, immunisation, screening for cervical and breast cancer and the efficacy of the new statin drugs for lowering plasma cholesterol. As Sir Denis Pereira Gray commented 'The challenge for this conference is how do we package the research evidence about nutrition so that throughtful family physicians will be convinced'.

'Family doctors and their patients: is effective nutrition possible?' Yes, but... The workshop discovered that the time barrier is not as absolute as it first seemed and there are ways of helping patients' motivation. It seems that the biggest need, before we seen more effective nutrition education in general practice, is for collection of clearer data about the benefits of nutrition and its provision in GP training courses, in continuing medical education and in software that the GP can share in his personal computer with the patient.

We want to thank Wiebe Visser, Director of the Dutch Dairy Foundation on Nutrition and Health for his sustained belief in the value of these workshops, for his enthusiastic assistance and for the financial support provided by this foundation. 